\title{
Deep Reinforcement Learning Based Massive Access Management for Ultra-Reliable Low-Latency Communications
}

\author{
Helin Yang, Student Member, IEEE, Zehui Xiong, Student Member, IEEE, Jun Zhao, Member, IEEE, \\ Dusit Niyato, Fellow, IEEE, Chau Yuen, Senior Member, IEEE, and Ruilong Deng, Senior Member, IEEE
}

\begin{abstract}
With the rapid deployment of the Internet of Things (IoT), fifth-generation (5G) and beyond 5G networks are required to support massive access of a huge number of devices over limited radio spectrum radio. In wireless networks, different devices have various quality-of-service $(\mathrm{QoS})$ requirements, ranging from ultra-reliable low latency communications (URLLC) to high transmission data rates. In this context, we present a joint energy-efficient subchannel assignment and power control approach to manage massive access requests while maximizing network energy efficiency (EE) and guaranteeing different QoS requirements. The latency constraint is transformed into a data rate constraint which makes the optimization problem tractable before modelling it as a multi-agent reinforcement learning problem. A distributed cooperative massive access approach based on deep reinforcement learning (DRL) is proposed to address the problem while meeting both reliability and latency constraints on URLLC services in massive access scenario. In addition, transfer learning and cooperative learning mechanisms are employed to enable communication links to work cooperatively in a distributed manner, which enhances the network performance and access success probability. Simulation results clearly show that the proposed distributed cooperative learning approach outperforms other existing approaches in terms of
\end{abstract}

This research is supported by Nanyang Technological University (NTU) Startup Grant, Alibaba-NTU Singapore Joint Research Institute (JRI), Singapore Ministry of Education Academic Research Fund Tier 1 RG128/18, Tier 1 RG115/19, Tier 1 RT07/19, Tier 1 RT01/19, and Tier 2 MOE2019-T21-176, NTU-WASP Joint Project, Singapore National Research Foundation under its Strategic Capability Research Centres Funding Initiative: Strategic Centre for Research in Privacy-Preserving Technologies \& Systems, Energy Research Institute @NTU, Singapore NRF National Satellite of Excellence, Design Science and Technology for Secure Critical Infrastructure NSoE DeSTSCI2019-0012, AI Singapore 100 Experiments (100E) programme, NTU Project for Large Vertical Take-Off \& Landing Research Platform, National Research Foundation (NRF), Singapore, under Singapore Energy Market Authority (EMA), Energy Resilience, NRF2017EWT-EP003-041, Singapore NRF2015-NRF-ISF001-2277, Singapore NRF National Satellite of Excellence, Design Science and Technology for Secure Critical Infrastructure NSoE DeST-SCI2019-0007, A*STAR-NTU-SUTD Joint Research Grant on Artificial Intelligence for the Future of Manufacturing RGANS1906, Wallenberg AI, Autonomous Systems and Software Program and Nanyang Technological University (WASP/NTU) under grant M4082187 (4080), Singapore Ministry of Education (MOE) Tier 1 (RG16/20), NTU-WeBank Joint Research Institute (JRI) (NWJ-2020-004), and Alibaba Group through Alibaba Innovative Research (AIR) Program. (Corresponding author: Jun Zhao.)

H. Yang, Z. Xiong, J. Zhao, and D. Niyato are with the School of Computer Science and Engineering, Nanyang Technological University, Singapore 639798 (e-mail: hyang013@e.ntu.edu.sg, zxiong002@e.ntu.edu.sg, junzhao@ntu.edu.sg,dniyato@ntu.edu.sg).

C. Yuen is with Singapore University of Technology and Design, Singapore 138682 (e-mail: yuenchau@sutd.edu.sg).

R. Deng is with the College of Control Science and Engineering, Zhejiang University, Hangzhou 310027, China, where he is also with the School of Cyber Science and Technology (e-mail: dengruilong@zju.edu.cn). meeting EE and improving the transmission success probability in massive access scenario.

Index Terms-Wireless networks, massive access, URLLC, spectrum and power management, multi-agent reinforcement learning, deep distributed cooperative learning.

\section{INTRODUCTION}

$\mathbf{W}$ ITH the rapid development of the Internet of Things (IoT), more and more IoT devices access wireless networks to support diverse applications, e.g., smart city, intelligent transportation, smart industry and healthcare (eHealth) [1], [2]. It can be predicted that the number of IoT devices will exceed 20 billion in 2020 and reach hundreds of billions in 2030 [1]. In this context, the upcoming fifth generation (5G) and beyond 5G (B5G) networks are required to provide seamless access and diverse services for massive IoT devices. Due to massive access of a large number of devices over limited radio spectrum, the deluge of spectrum access requests may lead to severe congestion with low transmission success probability [1], [3]. Considering the explosive increase in the number of devices, it is essential to improve the access efficiency in $5 \mathrm{G}$ networks for accommodating massive access with various quality-of-service (QoS) guarantees. For QoS guarantee, in $5 \mathrm{G}$ networks, ultra-reliable and low latency communications (URLLC) is one of the most challenging services with stringent low latency and high reliability requirements, i.e., in 3GPP, a general URLLC requirement of a one-way radio is $99.999 \%$ target reliability with $1 \mathrm{~ms}$ latency [4]. Consequently, URLLC entails great difficulty in massive access in $5 \mathrm{G}$ and $\mathrm{B} 5 \mathrm{G}$ wireless networks.

To relieve the radio access network congestion resulted from massive access, one of the simplest spectrum access schemes, termed random access procedure, was widely investigated recently. So far, there has been lots of research on massive random access for massive machine-type communications (mMTC), IoT networks and machine-to-machine (M2M) communications [5]-[12]. The authors in [5] and [6] proposed their contention-based random access models to enhance the access success probability and reduce the transmission delay. In [7], a two-stage random-access-based massive IoT uplink transmission protocol was presented to deal with the congestion caused by mMTC devices. Liu et al. in [8] investigated a priority-based multiple access protocol to ensure the fairness of different devices. Furthermore, grant-based and grant-free are two common random access schemes which can provide 
devices' access statuses after processing device detection and channel estimation [3], [9], [10], but accurate channel state information (CSI) requirements of a massive number of devices may be impractical. Besides, several methods were presented to enhance the traditional random access performance, such as access class barring (ACB), slotted access, and backoff [11], [12]. For instance, in [12], an efficient random access procedure based on ACB was investigated to decrease the access delay and the power consumption when wireless networks have a congestion problem resulted from massive access.

The aforementioned approaches [5]-[12] based on random access are simple, flexible and could be applied without central coordinator with massive wireless connections. However, these proposals achieve limited improvement and high access failures which remain the performance bottleneck for massive access. In particular, the high transmission success probability is not easily guaranteed when the devices have strict URLLC requirements. To satisfy the critical requirements of URLLC in massive IoT or mMTC, many studies have presented the advanced spectrum access schemes [13]-[19]. Weerasinghe et al. proposed a priority-based massive access approach to support reliable and low latency access for mMTC devices [13], where devices are categorized into a number of groups with different priority access levels. A probability density function of signal-to-noise ratio (SNR) was derived for a large number of uplink URLLC devices in [14], and numerical results verified that the presented model can satisfy the critical requirements of URLLC. Popovski et al. in [15] discussed the principles of wireless access for URLLC and provided a perspective on the relationship between latency, packet size and bandwidth. In [16] and [17], grant-free spectrum access was adopted to reduce transmission latency as well as improve the spectrum utilization in URLLC scenario. In [18] and [19], different resource management schemes were developed to shown how to update the system parameters that allow meeting the URLLC requirements in industrial IoT networks, since industrial automation requires strict low latency and high reliability for manufacturing control. Nevertheless, only a few literatures [13] and [14] investigated how to meet strict URRLC requirements in massive access scenario, and the optimization objective of these two studies are just a single time slot optimization problem, where the massive access decision approaches may converge to the sub-optimal solution and obtain the greedy-search like performance due to the ignorance of the historical network state and the long term benefit.

\section{A. Related Works}

Recently, several emerging technologies of $5 \mathrm{G}$, i.e., massive multiple-input multiple-output (MIMO), non-orthogonal multiple access (NOMA) and device-to-device (D2D) communications are applied to support massive connectivity over limited available radio resources. Chen et al. in [12] and [20] presented non-orthogonal communication frameworks based on massive NOMA to support massive connections, and the transmit power values were optimized to mitigate severe co-channel interference by using interference cancellation techniques [21]. In addition, an application-specific NOMAbased communication architecture was investigated for future URLLC Tactile Internet [22]. In [14], [15], [23] and [24], the authors presented coordinated and uncoordinated access protocols to support massive connectivity in massive MIMO systems by exploiting large spatial degrees of freedom to admit massive IoT devices. Specifically, the authors in [14] and [15] discussed that the massive MIMO system can be acted as a natural enabler for URLLC where multiple antenna systems can support high capacity, spatial multiplexing and diversity links. Moreover, a potential solution for the massive access congestion problem is to offload the large amount of traffic onto D2D communication links [25], [26], which can directly reduce devices' energy consumption and transmission delay, as well as improve spectrum efficiency. D2D-based transmission protocols for supporting URLLC services were proposed in [27] and [28], where devices are classified into a number of groups based on their QoS requirements, i.e., stringent low latency and high reliability requirements, with radio resources allocated accordingly.

In addition, energy efficiency (EE) plays an important role in green wireless networks. The reasons are that most of devices (e.g., sensors, actuators and wearable devices) are power constrained and energy consumption is massive and expensive under high-density scenario of devices. In [29] and [30], the authors optimized the joint radio access and power resource allocation to maximize $\mathrm{EE}$ while guaranteeing the transmission delay requirements and transmit power constraints of a huge number of devices. To mitigate co-channel interference and further enhance the EE performance of NOMA-based systems with massive IoT devices, subchannel allocation and power control approaches were proposed in [19], [29], [31]. Furthermore, Miao et al. [32] proposed an energy-efficient clustering scheme to address spectrum access problem for massive M2M communications. Although the authors in [29]-[32] mainly focused on the EE maximization based massive access, the different QoS requirements (such as latency and reliability) of devices has not been well studied in massive access scenario.

Considering that intelligence is an important characteristic of future wireless networks, many studies have investigated application of reinforcement learning (RL) in the field of massive access management recently [9], [23], [33]-[42]. Different distributed RL frameworks were proposed to address the massive access management problem under massive scale and stringent resource constraints [33], [34], where each device has the ability to intelligently make its informed transmission decision by itself without central controller. The authors in [9] and [23] adopted the sparse dictionary learning to facilitate massive connectivity for a massive-device multiple access communication system, and the learning structure does not need any prior knowledge of active devices. Furthermore, the delay-aware access control of massive random access for mMTC and M2M was studied in [33], [35] and [36], and spectrum access algorithms based on RL were proposed to determine the access decision with high successful connections and low network access latency. As future wireless networks are complex and large-scale, RL cannot effectivity deal with the high-dimensional input state space, deep reinforcement 
learning (DRL) (DRL combines deep learning with RL to learn itself from experience) was developed to solve complex spectrum access decision-making tasks under large-state space [37]-[42]. The authors in [37]-[39] proposed distributed dynamic spectrum access (DSA) approaches based on DRL to search the optimal solution for the DSA problem under the large-state space and local observation information. These distributed learning approaches are capable of encouraging devices to make spectrum access decisions according to their own observations without central controller, and hence they have a great potential for finding efficient solutions for realtime services. Hua et al. in [40] presented a network-powered deep distributional Q-network to allocate radio resources for diversified services in 5G networks. Moreover, Yu et al. in [41] investigated a DRL-based multiple access protocol to learn the optimal spectrum access policy with considering service fairness, and Mohammadi et al. in [42] employed a deep Q-network (DQN) algorithm for cognitive radio underlay DSA which outperforms the distributed multi-resource allocation. However, the above works [37]-[42] did not investigate how to address the massive access management problem in their presented spectrum access approaches based on DRL, and most of the works did not consider the stringent reliability and latency constraints into the optimization problem.

\section{B. Contributions}

Motivated by the above analysis and observations, in order to address the above mentioned challenges in massive access for $5 \mathrm{G}$ and $\mathrm{B} 5 \mathrm{G}$ wireless networks, this paper not only studies on how to manage the massive access requests from a huge number of devices, but also takes various QoS requirements (ranging from strict low latency and high reliability to minimum data rate) into consideration. Besides, a novel distributed cooperative learning approach based QoS-aware massive access is presented to optimize the joint subchannel assignment and transmission power control strategy without a centralized controller. The main contributions of the paper are summarized as follows:

- We formulate a joint subchannel assignment and transmission power control problem for massive access considering different practical QoS requirements, and the energy-efficient massive access management problem is modelled as a multi-agent RL problem. Hence, each device has the ability to intelligently make its spectrum access decision according to its own instantaneous observations.

- A distributed cooperative subchannel assignment and transmission power control approach based on DRL is proposed for the first time to guarantee both the strict reliability and latency requirements on URLLC services in massive access scenario, where the latency constraint is transformed into a data rate constraint which can make the optimization problem tractable. Specifically, a proper QoS-aware reward function is built to cover both the network EE and devices' QoS requirements into the learning process.

- In addition, we apply transfer learning and cooperative learning mechanisms to enable communication links to

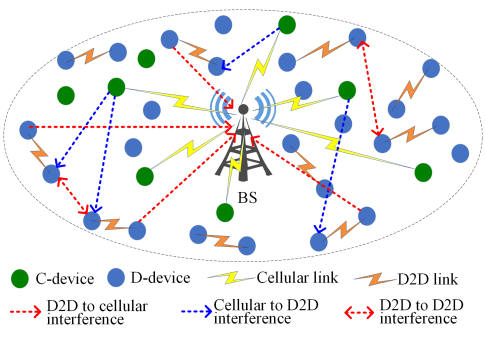

Fig. 1. System model of the massive-device network.

work cooperatively in a distributed cooperative manner, in order to improve the network performance and transmission success probability based on local observation information. In detail, in transfer learning, if a new device joins the network or applies a new service, or one communication link achieves poor performance (e.g, low QoS satisfaction level or low convergence speed), then it can directly search the expert agent from the neighbors, and utilizes the transfer learning model from the expert agent instead of building a new learning model. In cooperative learning, devices are encouraged to share their selected actions with their neighbors and take turns to make decisions, which can enhance the overall benefit by choosing the actions jointly instead of independently.

- Extensive simulation results are presented to verify the effectiveness of the proposed distributed cooperative learning approach in massive access scenario, and demonstrate the superiority of the proposed learning approach in terms of meeting the network EE and improving the transmission success probability compared with other existing approaches.

The rest of this paper is organized as follows. In Section II, the system model and problem formulation are provided. The massive access management problem is modelled as a Markov decision making process in Section III. Section IV proposes a distributed cooperative multi-agent learning based massive access approach. Section V provides simulation results and Section VI concludes the paper.

\section{System Model and Problem Formulation}

We consider a wireless network, as shown in Fig. 1, which consists of a base station (BS) at the center and a massive number of devices with each device being equipped with a single antenna. The devices are mainly divided into two types: cellular devices (denoted C-device) which communicate with the BS over the orthogonal spectrum subchannels, and D2D devices (D-device) which establish D2D communication links if two of them want to communicate with each other and they are close enough. In the network, D-devices can opportunistically access subchannels of C-devices while ensuring that the generated interference from D2D pairs to C-devices should not affect the QoS requirements of C-devices. We assume that each $\mathrm{C}$-device can be allocated with multiple subchannels, and each subchannel only serve for at most one C-device in one time slot. In addition, each D2D pair can share multiple subchannels of C-devices. 
Let $K, M$ and $N$ denote the number of C-devices, D2D pairs and subchannels, respectively. The sets of corresponding C-device, D2D pair and subchannel are denoted by $\mathcal{K}=$ $\{1,2, \ldots, K\}, \mathcal{M}=\{1,2, \ldots, M\}$ and $\mathcal{N}=\{1,2, \ldots, N\}$, respectively. Let $Z$ denote the total number of communication links, $Z=K+M$, and its corresponding communication link set is defined by $\mathcal{Z}=\{1,2, \ldots, Z\}$. Denote by $h_{k}$ and $h_{m}$ the channel coefficients of the desired transmission links from the $k$-th $\mathrm{C}$-device to the $\mathrm{BS}$, and the transmitter to the receiver in the $m$-th D2D pair, respectively. Denote by $g_{k, m}, g_{m, B}$ and $g_{m^{\prime}, m}$ the interference channel gains from the $k$-th C-device to the receiver of D2D pair $m$, the transmitter of D2D pair $m$ to the BS, and the transmitter of the $m^{\prime}$-th D2D pair to the receiver of the $m$-th $\mathrm{D} 2 \mathrm{D}$ pair, respectively.

In the spectrum reusing case, C-devices suffer co-channel interference from the transmitters of D2D pairs if they share the subchannels with $\mathrm{D} 2 \mathrm{D}$ pairs. As a result, the received signal-to-interference-plus-noise ratio (SINR) at the BS for Cdevice $k$ on the $n$-th subchannel is expressed by

$$
S I N R_{k, n}^{\mathrm{c}}=\frac{P_{k, n}^{\mathrm{c}} h_{k}}{\sum_{m \in \mathcal{M}} \rho_{m, n} P_{m, n}^{\mathrm{d}} g_{m, B}+\delta_{k}^{2}},
$$

where $P_{m, n}^{\mathrm{c}}$ and $P_{m, n}^{\mathrm{d}}$ denote the transmission power values of the $k$-th C-device and the $m$-th D2D pair' transmitter on the $n$-th subchannel, respectively. $\rho_{m, n}$ is the subchannel access indicator, $\rho_{m, n} \in\{0,1\} ; \rho_{m, n}=1$ indicates that the $m$-th D2D pair assigns on the $n$-th subchannel; otherwise, $\rho_{m, n}=0 . \delta_{k}^{2}$ is the additive white Gaussian noise power. In (1), $\sum_{m \in \mathcal{M}} \rho_{m, n} P_{m, n}^{\mathrm{d}} g_{m, B}$ is the co-channel interference.

In addition, subchannel sharing also leads to the co-channel interference to D2D pairs, which is the generated interference from the co-channel C-device and co-channel D2D pairs on the same subchannel. Hence, the received SINR at the $m$-th D2D pair's receiver when it reuses the $n$-th subchannel of the $k$-th $\mathrm{C}$-device is given by

$$
\begin{aligned}
& S I N R_{m, n}^{\mathrm{d}} \\
& =\frac{P_{m, n}^{\mathrm{d}} h_{m}}{P_{k, n}^{\mathrm{c}} g_{k, m}+\sum_{m^{\prime} \in \mathcal{M}, m^{\prime} \neq m} \rho_{m, m^{\prime}, n} P_{m^{\prime}, n}^{\mathrm{d}} g_{m^{\prime}, m}+\delta_{m}^{2}},
\end{aligned}
$$

where $\rho_{m, m^{\prime}, n}$ is the subchannel access indicator, $\rho_{m, m^{\prime}, n} \in$ $\{0,1\} ; \rho_{m, m^{\prime}, n}=1$ indicates that both the $m$-th D2D pair and $m^{\prime}$-th D2D pair assign on the same $n$-th subchannel in one time slot; otherwise, $\rho_{m, m^{\prime}, n}=0 . \delta_{m}^{2}$ is the additive white Gaussian noise power.

Then, the data rate of the $k$-th $\mathrm{C}$-device and the $m$-th D2D pair on their assigned subchannels are respectively expressed by

$$
R_{k}^{\mathrm{c}}=\sum_{n \in \mathcal{N}} \rho_{k, n} \log _{2}\left(1+S I N R_{k, n}^{\mathrm{c}}\right),
$$

and

$$
R_{m}^{\mathrm{d}}=\sum_{n \in \mathcal{N}} \rho_{m, n} \log _{2}\left(1+S I N R_{m, n}^{\mathrm{d}}\right) .
$$

where $\rho_{k, n}$ is the subchannel access indicator which has the same definition of $\rho_{m, n}$ and $\rho_{m, m^{\prime}, n}$ as aforementioned above.

\section{A. Network Requirements}

1) URLLC Requirements: In 5G and B5G networks, different devices have different QoS requirements, i.e., some devices have ultra-high reliability communication requirements, some devices need strict low-latency services, and even some devices have both the stringent low latency and high reliability requirements. For example, intelligent transportation and factory automation have stringent URLLC requirements for real-time safety information exchange or hazard monitoring, where the maximum latency is less than $5 \mathrm{~ms}$ (even about 0.1 ms) and the transmission reliability needs to be higher than that $1-10^{-5}$ (or even $1-10^{-5}$ ), but they do not need the high data rate.

For URLLC requirements, we assume that the packet arrival process of the $i$-th $(i \in \mathcal{Z})$ communication link is independent and identically distributed and follows Poisson distribution with the arrival rate $\lambda_{i}$ [11], [19]. Let $L_{i}^{\text {packet }}$ denote the packet size in bits of the $i$-th communication link, and it follows the exponential distribution with mean packet size $\bar{L}_{i}^{\text {packet }}$. Generally, the total latency mainly includes the transmission delay $\left(T_{\mathrm{tr}}\right)$, queuing waiting delay $\left(T_{\mathrm{qw}}\right)$ and processing/computing delay $\left(T_{\mathrm{pc}}\right)$, which can be expressed by [19]

$$
T_{\text {Latency }}=T_{\mathrm{tr}}+T_{\mathrm{qw}}+T_{\mathrm{pc}} .
$$

In (5), the transmission delay of the packet $L_{i}^{\text {packet }}$ can be given by $T_{\mathrm{tr}}=L_{i}^{\text {packet }} /\left(W \times R_{i}\right)$, where $W$ is the bandwidth of each subchannel and $R_{i}$ is the data rate given in (3) or (4), respectively.

Due to the low latency constraint, each packet requires to be successfully transmitted in a given time period. Let $T_{\max }$ denote the maximum tolerable latency threshold, so the latency outage probability of URLLC can be given by

$$
p_{i}^{\text {Latency }}=\operatorname{Pr}\left\{T_{\text {Latency }}>T_{\max }\right\} \leq p_{\max }^{\text {Latency }},
$$

where $p_{\max }^{\text {Latency }}$ is the maximum SINR violation probability.

It is hard to directly calculate the device's packet latency shown in (5), and hence the outrage probability in (6) is difficult to be achieved. However, we can transform the latency constraint (6) into the data rate constraint by using max-plus queuing methods [43].

To guarantee the latency outage probability constraint shown in (6), the data rate $R_{i}^{\text {URLLC }}$ of each URLLC service of the $i$-th communication link should meet

$$
R_{i}^{\mathrm{URLLC}} \geq \frac{\bar{L}_{i}^{\text {packet }}}{W T_{\max }}\left[F_{i}-f_{-1}\left(p_{\max }^{\mathrm{Lat}} F_{i} e^{F_{i}}\right)\right] \triangleq R_{i, \text { min }}^{\mathrm{URLC}},
$$

where $\left.f_{-1}(\cdot):\left[-e^{-1}, 0\right) \rightarrow[-1, \infty)\right]$ denotes the lower branch of Lambert function meeting $y=f_{-1}\left(y e^{y}\right)$ [43], $F_{i}=\lambda_{i} T_{\max } /\left(1-e^{\lambda_{i} T_{\max }}\right)$, and $R_{i, \min }^{\mathrm{URLC}}$ is the minimum data rate to ensure the latency constraint shown in (6). The relevant proof of (7) can be seen in [43, Th. 2]. If the transmission data rate is less than the minimum data rate threshold, in other words, the transmission latency exceeds the maximum latency threshold, the current URLLC service is unsuccessful and its corresponding packet transmission is stopped. 
In addition, the SINR value can be used to characterize the reliability of URLLC. In detail, the received SINR at the receiver should be beyond the minimum SINR threshold. Otherwise, the received signal cannot be successfully demodulated. Hence, the outage probability in term of SINR can be given by

$$
p_{i, n}^{\text {outage }}=\operatorname{Pr}\left\{S I N R_{i, n}<S I N R_{i, n}^{\min }\right\} \leq p_{\max }^{\text {outage }},
$$

where $S I N R_{i, n}^{\min }$ denotes the minimum SINR threshold of communication link $i$ on the $n$-th subchannel and $p_{\max }^{\text {outage }}$ denotes the maximum violation probability.

2) Minimum Data Rate Requirements: In addition to the high reliability and low latency requirements mentioned in Section, some C-devices and D2D pairs may have the minimum data rate requirements. Let $R_{k, \min }^{\mathrm{c}}$ and $R_{m, \min }^{\mathrm{d}}$ denote the minimum data rate requirements of the $k$-th $\mathrm{C}$-device and the $m$-th $\mathrm{D} 2 \mathrm{D}$ pair, respectively. Then, the minimum data rate requirements are given by

$$
R_{k}^{\mathrm{c}} \geq R_{k, \min }^{\mathrm{c}}, \forall k ; \quad R_{m}^{\mathrm{d}} \geq R_{m, \min }^{\mathrm{d}}, \forall m .
$$

\section{B. Problem Formulation}

The objective of this paper is to maximize the overall network $\mathrm{EE}$ ( $\mathrm{EE}$ is the ratio of the sum data rate and the sum energy consumption) while guaranteeing the network requirements shown in Section III.A. Then, the massive access management problem (joint subchannel access and transmission power control) is formulated as follows:

$$
\begin{array}{ll}
\max _{\boldsymbol{\rho}, \boldsymbol{P}} & \eta_{E E}=\frac{\sum_{k \in \mathcal{K}} R_{k}^{\mathrm{c}}+\sum_{m \in \mathcal{M}} R_{m}^{\mathrm{d}}}{\sum_{n \in \mathcal{N}}\left(\sum_{k \in \mathcal{K}} \rho_{k, n} P_{k, n}^{\mathrm{c}}+\sum_{m \in \mathcal{M}} \rho_{m, n} P_{m, n}^{\mathrm{d}}\right)+Z P_{c i r}} \\
\text { s.t. } & \text { (a) : }(7),(8),(9) ; \\
& \text { (b) : } \rho_{k, n} \in\{0,1\}, \rho_{m, n} \in\{0,1\}, \forall k, m, n ; \\
& \text { (c) : } \sum_{k \in \mathcal{K}} \rho_{n, k} \leq 1, \forall n \in \mathcal{N} ; \\
& \text { (d) }: \sum_{n \in \mathcal{N}} \rho_{k, n} P_{k, n}^{\mathrm{c}} \leq P_{\max }^{\mathrm{c}}, \forall k \in \mathcal{K} ; \\
& \text { (e) }: \sum_{n \in \mathcal{N}} \rho_{m, n} P_{m, n}^{\mathrm{d}} \leq P_{\max }^{\mathrm{d}}, \forall m \in \mathcal{M},
\end{array}
$$

where $\boldsymbol{\rho}$ and $\boldsymbol{P}$ denote the subchannel assignment and power control strategies, respectively. $P_{\max }^{\mathrm{c}}$ and $P_{\max }^{\mathrm{d}}$ denote the maximum transmission power values of each $\mathrm{C}$-device and each D-device, respectively. $P_{c i r}$ denotes the circuit power consumption of one communication link. Constraint (10c) guarantees that each subchannel is allocated at most one Cdevice. Constraint (10d) and (10e) are imposed to ensure that the power constraints of devices.

\section{Problem TRAnSformation}

Clearly, the optimization problem given in (10) is not easy to be solved as it is a non-convex combination and NP-hard problem. More importantly, the optimization objective is just a single time slot optimization problem, where the massive access decision is only based on the current state with the fixed optimization function. The single time slot massive access decision approaches may converge to the suboptimal solution and obtain the greedy-search like performance due to the lack of the historical network state and the long term benefit.
Hence, in this section, model-free RL as a dynamic programming tool can be applied to address the decision-making problem by learning the optimal solutions over dynamic environment. Similar to most of existing studies [32]-[42], we apply Markov Decision Process (MDP) to model the massive access decision-making problem in the RL framework by transforming the optimization problem (10) into MDP.

In the MDP model, each communication link acts as an agent by interacting with outside environment and the MDP model is defined as a tuple $(\mathcal{S}, \mathcal{A}, \mathcal{P}, r, \gamma)$, where $\mathcal{S}$ is the state space set, $\mathcal{A}$ denotes the action space set, $\mathcal{P}$ indicates the transition probability: $\mathcal{P}\left(s_{t+1} \mid s_{t}, a_{t}\right)$ is the probability of transferring from a current state $s_{t} \in \mathcal{S}$ to a new state $s_{t+1} \in \mathcal{S}$ after taking an action $a_{t} \in \mathcal{A}, r$ denotes the immediate reward, and $\gamma \in(0,1)$ denotes the discount factor. The details of the MDP model for massive access management are presented as follows.

State: In 5G and B5G networks, the network state is defined as $s=\left\{s_{\text {cha }}, s_{\text {cq }}, s_{\text {tr }}, s_{\mathrm{QoS}}\right\} \in \mathcal{S}, s_{\text {cha }}$ indicates the subchannel working status (idle or busy); $s_{\mathrm{cq}}$ depicts the channel quality (i.e., SINR); $s_{\text {tr }}$ is the traffic load of each packet; and $s_{\mathrm{QoS}}$ represents the QoS satisfaction level (the transmission success probability), such as the satisfaction levels of the minimum data rate, latency and reliability.

Action: For the massive access management problem, each agent will decide which subchannels can be assigned and how much transmit power should be allocated on the assigned subchannels. Hence, the action can be defined as $a=\left\{\rho_{\text {cha }}, P_{\text {pow }}\right\} \in \mathcal{A}$ which includes the subchannel assignment indicator $\left(\rho_{\text {cha }}\right)$ and the transmission power $\left(P_{\text {pow }}\right)$. At each time slot, the action of each device consists of channel assignment indicator $\rho_{\text {cha }} \in\{0,1\}$ and transmission power level $P_{\text {pow }} \in\{50,150,300,500\}$ in $\mathrm{mW}$ where the transmission power is discretized into four levels. We can observe that the action space of each device is not big, but the overall action space of all devices in the massive access scenario is large. Hence, we need to discretize the transmission power numbers as small as possible, so the four transmission power levels are chosen in this paper instead of the higher number of transmission power levels.

Reward function: In order to reflect the device experience which the network wants to optimize, RL requires designing the specific reward function where the learning process is generally driven by the reward. In RL, each agent searches its decision-making policy by maximizing its reward under the interaction with environment. Hence, it is important to design an efficient reward function to improve the devices' service satisfaction levels.

Here, let $\mathcal{Z}^{\prime}$ denotes the set of communication links in the URLLC scenario where the devices have both the reliability and latency requirements, and $\mathcal{Z}^{\prime \prime}$ denotes the set of communication links in the normal scenario where the devices have minimum data requirements. $\left|\mathcal{Z}^{\prime}\right|=Z^{\prime}$ and $\left|\mathcal{Z}^{\prime \prime}\right|=Z^{\prime \prime}$. Let $R_{i}^{\text {nor }}$ and $R_{i \text { min }}^{\text {nor }}$ denote the instantaneous data rate and the minimum data rate threshold in the normal scenario, respectively.

According the optimization problem shown in (10), considering the different QoS requirements, we design a new QoS- 
aware reward function for the massive access management problem, where the reward function of the $i$-th communication link includes the network EE, as well as the reliability, latency and minimum data rate requirements, which is expressed by

$$
r_{i}=\eta_{i, E E}-c_{1} \chi_{i}^{\mathrm{URLLC}}-c_{2} \chi_{i}^{\mathrm{nor}}
$$

where

$$
\chi_{i}^{\mathrm{URLLC}}=\left\{\begin{array}{l}
1, \text { if }(7) \text { or }(8) \text { is not satisfied }, \\
0, \text { otherwise }
\end{array}\right.
$$

and

$$
\chi_{i}^{\text {nor }}=\left\{\begin{array}{l}
1, \text { if } R_{i}^{\text {nor }}<R_{i, \min }^{\text {nor }} \\
0, \text { otherwise }
\end{array}\right.
$$

In (11), the part 1 indicates the immediate utility (network $\mathrm{EE})$, the part 2 and part 3 are the cost functions of the transmission failures which are defined as the unsatisfied URLLC requirements and the unsatisfied minimum data rate requirements, respectively. The parameters $c_{i}, i \in\{1,2\}$ denote the positive constants of the latter two parts in (11) and they are adopted for balancing the utility and cost [19], [28], [39].

The objectives of (12) and (13) are to refract the QoS satisfaction levels of both the URLLC services and normal services, respectively. In detail, if the URLLC requirement of one packet is satisfied in the current time slot, then $\chi_{i}^{\mathrm{URLLC}}=0$; if the minimum data rate is satisfied, then $\chi_{i}^{\text {nor }}=0$. This means that there is no cost or punishment of the reward due to the successful transmission with QoS guarantees. Otherwise, $\chi_{i}^{\mathrm{URLLC}}=1$, or $\chi_{i}^{\text {nor }}=1$.

The reward function shown in (11) may have the same reward values for some cases. For example, the following two cases may have the same reward for different values: Case I, the URLLC requirement is not satisfied while the minimum data rate requirement is satisfied, then $\chi^{\mathrm{URLLC}}=1$ and $\chi^{\text {nor }}=0$; Case II, the URLLC requirement is satisfied while the minimum data rate requirement is not satisfied, then $\chi^{\mathrm{URLLC}}=0$ and $\chi^{\text {nor }}=1$. For these two cases, they may have the same reward function values: $r=\eta_{E E}-c_{1} * 1-c_{2} * 0$ and $r=\eta_{E E}-c_{1} * 0-c_{2} * 1$ with $c_{1}=c_{2}$ being the punishment factors. If the punishment factors $c_{1} \neq c_{2}$, the two cases have different reward function values. We would like to mention that the values of the punishment factors $c_{1}$ and $c_{2}$ have important impacts on the reward function, if $c_{1}>c_{2}$, the URLLC requirement has the higher impact on the final reward value than that of the minimum data rate requirement; by contract, if $c_{1}<c_{2}$, the minimum data rate requirement has the higher impact on the final reward value than that of the URLLC requirement. Furthermore, if $c_{1}=c_{2}$, both the URLLC requirement and minimum data rate requirement have the same impacts on the reward value.

In RL, each agent in the MPD model tries to select a policy $\pi$ to maximize a discounted accumulative reward, where $\pi$ is a mapping from state $s$ with the probability distribution over actions that the agent can take: $\pi(s): \mathcal{S} \rightarrow \mathcal{A}$. The discounted accumulative reward is also a called the state-value function for starting the state $s$ with the current policy $\pi$, and it is defined by

$$
V^{\pi}(s)=\left\{\sum_{t=1}^{\infty} \gamma^{t} r_{t}\left(s_{t}, a_{t}\right) \mid s_{0}=s, \pi\right\} .
$$

The function $V^{\pi}(s)$ in (14) is usually applied to test the quality of the selected policy $\pi$ when the agent selects the action $a$. The MPD model tries to search the optimal statevalue function $V^{*}(s)$, which is expressed by

$$
V^{*}(s)=\max _{\pi} V^{\pi}(s) .
$$

Once $V^{*}(s)$ is achieved, the optimal policy $\pi^{*}\left(s_{t}\right)$ under the current state $s_{t}$ is determined by

$$
\pi^{*}\left(s_{t}\right)=\arg \max _{a_{t} \in \mathcal{A}} \bar{U}_{t}\left(s_{t}, a_{t}\right)+\sum_{s_{t+1}} P\left(s_{t+1} \mid s_{t}, a_{t}\right) V^{*}\left(s_{t+1}\right),
$$

where $\bar{U}_{t}\left(s_{t}, a_{t}\right)$ denotes the expected reward by selecting action $a_{t}$ at state $s_{t}$. To calculate $V^{*}(s)$, the iterative algorithms can be applied. However, it is difficult to get the transition probability $P\left(s_{t+1} \mid s_{t}, a_{t}\right)$ in practical environments, but RL algorithms, such as Q-learning, policy gradient and DQN, are widely employed to address MDP problems under environment uncertainty.

In Q-learning algorithm, the Q-function is used to calculate the accumulative reward for starting from a state $s$ by taking an action $a$ with the selected policy $\pi$, which can be given by

$$
Q^{\pi}(s, a)=\left\{\sum_{t=1}^{\infty} \gamma^{t} r_{t}\left(s_{t}, a_{t}\right) \mid s_{0}=s, a_{0}=a, \pi\right\} .
$$

Similarly, the optimal Q-function is obtained by

$$
Q^{*}(s, a)=\max _{\pi} V^{\pi}(s, a) .
$$

In Q-learning algorithm, the Q-function is updated by

$$
\begin{aligned}
& Q_{t+1}\left(s_{t}, a_{t}\right)=Q_{t}\left(s_{t}, a_{t}\right) \\
& +\alpha\left[r_{t+1}+\gamma \max _{a_{t+1}} Q_{t}\left(s_{t+1}, a_{t+1}\right)-Q_{t}\left(s_{t}, a_{t}\right)\right],
\end{aligned}
$$

where $\alpha$ denotes the learning rate. When $Q^{*}(s, a)$ is achieved, the optimal policy is determine by

$$
\pi^{*}(s)=\arg \max _{a \in A} Q^{*}(s, a) .
$$

\section{Distributed Cooperative Multi-Agent RL BASED MASSIVE ACCESS}

Even through Q-learning is widely adopted to design the resource management policy in wireless networks without knowing the transition probability in advance, it has some key limitations for its application in large-scale 5G and B5G networks, such as Q-learning has slow convergence speed under large-state space, and it cannot deal with large continuous state-action spaces. Recently, a great potential is demonstrated by DRL that combines neural networks (NNs) with Q-learning, called DQN, which can efficiently address the above mentioned problems and achieve better performance owing to the following reasons. Firstly, DQN adopts NNs 
to map from the observed state to action between different layers, instead of using storage memory to store the Q-values. Secondly, large-scale models can be represented from high dimensional raw data by using NNs. Furthermore, applying experience replay and generalization capability brought by NNs, DQN can improve network performance.

In 5G and B5G networks shown in Fig. 1, massive communication links aim to access the limited radio spectrum, which can be modelled as a multi-agent RL problem, where each communication link is regarded as a learning agent to interact with network environment to learn its experience, and the learned experience is then utilized to optimize its own spectrum access strategy. Massive agents explore the outside network environment and search spectrum access and power control strategies according to the observations of the network state.

The proposed deep multi-agent RL based approach consists of two stages, a training stage and a distributed cooperative implementation stage. The main contributions of the proposed distributed cooperative multi-agent RL based approach for massive access are provided in the following two subsections in detail.

\section{A. Training Stage of Multi-Agent RL for Massive Access}

For the training sage, we adopt DQN with experience relay to train the multi-agent RL for efficient learning of massive access policies. Fig. 2 indicates the training process. All communication links are regarded as agents and the wireless network acts as the environment. Firstly, each agent intelligently observes its current state (e.g., subchannel status (busy or idle), channel quality, traffic load and QoS satisfaction levels) by integrating with the environment. Then, it makes decision and chooses one action according to its learned policy. After that, the environment feedbacks a new state and an immediate reward to each agent. Based on the feedback, all agents smartly learn new policies in the next time step. The optimal parameters of DQN can be trained with an infinite number of time steps. In addition, the experience replay mechanism is adopted to improve the learning speed, the learning efficiency and the learning stability toward the optimal policy for the massive access management. The training data is stored in the storage memory, and a random mini-batch data is sampled from the storage memory and used to optimize the weight of DQN.

At each training or learning step, each DQN agent updates its weight, $\boldsymbol{\theta}$, to minimize the loss function defined by

$$
\begin{aligned}
& \operatorname{Loss}\left(\boldsymbol{\theta}_{t}\right)= \\
& {\left[r_{t+1}\left(s_{t}, a_{t}\right)+\gamma \max _{a \in \mathcal{A}} Q_{t}\left(s_{t+1}, a_{t+1}, \boldsymbol{\theta}_{t}\right)-Q_{t}\left(s_{t}, a_{t}, \boldsymbol{\theta}_{t}\right)\right]^{2} .}
\end{aligned}
$$

One important reason of adopting DQN is to update the loss functions given in (21) at each tainting step to decrease the computational complexity for large-scale learning problems [37]-[42]. The DQN weight $\boldsymbol{\theta}$ is obtained by using the gradient descent method, which can be expressed as

$$
\boldsymbol{\theta}_{t+1}=\boldsymbol{\theta}_{t}+\beta \nabla \operatorname{Loss}\left(\boldsymbol{\theta}_{t}\right),
$$

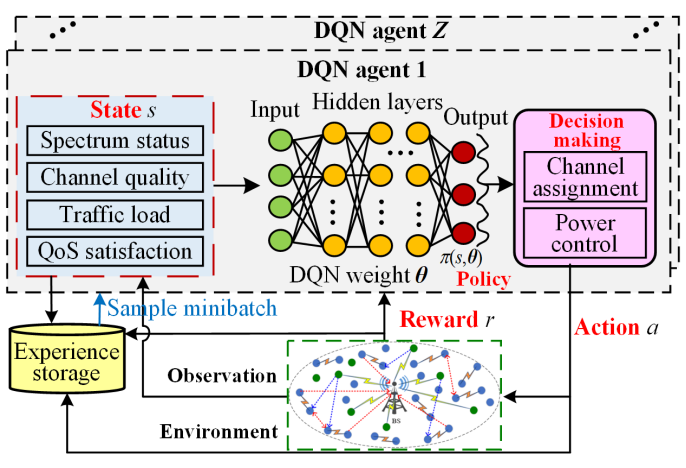

Fig. 2. DQN training based intelligent subchannel assignment and power control for massive access.

where $\beta$ denotes the learning rate of the weight $\boldsymbol{\theta}$, and $\nabla($. is the first-order partial derivative.

Then, each agent selects its action according to the selected policy $\pi\left(s_{t}, \boldsymbol{\theta}_{t}\right)$, which is given by

$$
\pi\left(s_{t}, \boldsymbol{\theta}_{t}\right)=\arg \max _{a \in \mathcal{A}}\left\{Q_{t}\left(s_{t}, a_{t}, \boldsymbol{\theta}_{t}\right)\right\} .
$$

Pseudocode for training DQN is presented in Algorithm 1. The communication environment contains both the C-devices and D-devices and their positions in the served coverage area of the BS, and the channel gains are generated based on their positions. Each agent has its trained DQN model that takes as input of current observed state $s_{t}$ and outputs the Q-function with the selected action $a_{t}$. The training loop has a finite number of episodes $N^{\text {epi }}$ (i.e., tasks) and each episode has $T$ training iterations. At each training step, after observing the current state $s_{t}$, all agents explore the state-action space by applying the $\varepsilon$-greedy method, where each action $a_{t}$ is randomly selected with the probability $\varepsilon_{t}$ while the action is chosen with the largest Q-value $Q_{t}\left(s_{t}, a_{t}, \boldsymbol{\theta}_{t}\right)$ with the probability $1-\varepsilon_{t}$. After executing $a_{t}$ (subchannel assignment and power control), agents will receive an immediate reward $r_{t}$ and observe a new state $s_{t+1}$ from the environment. Then, the experience $e_{t}=\left(s_{t}, a_{t}, r\left(s_{t}, a_{t}\right), s_{t+1}\right)$ is stored into the replay memory $D$. At each episode, a mini-batch data from the memory is sampled to update the weight $\boldsymbol{\theta}_{t}$ of DQN.

\section{B. Distributed Cooperative Implementation of Multi-Agent RL for Massive Access}

The above mentioned trained DQN models with the computation intensive training procedure are shown in Section IV.A, which can be completed offline at BS since BS has powerful computing capacity to train large-scale models. After adequate training, the trained models are utilized for implementation. In this subsection, we propose a distributed cooperative learning approach to optimize the network performance in massive access scenario.

During the distributed cooperative implementation stage, at each learning step, each communication link (agent) utilizes its local observation and information to choose its action with the maximum Q-value. In this case, each agent has no knowledge of actions chosen by other agents if the actions are updated simultaneously and new joined agents need to train 


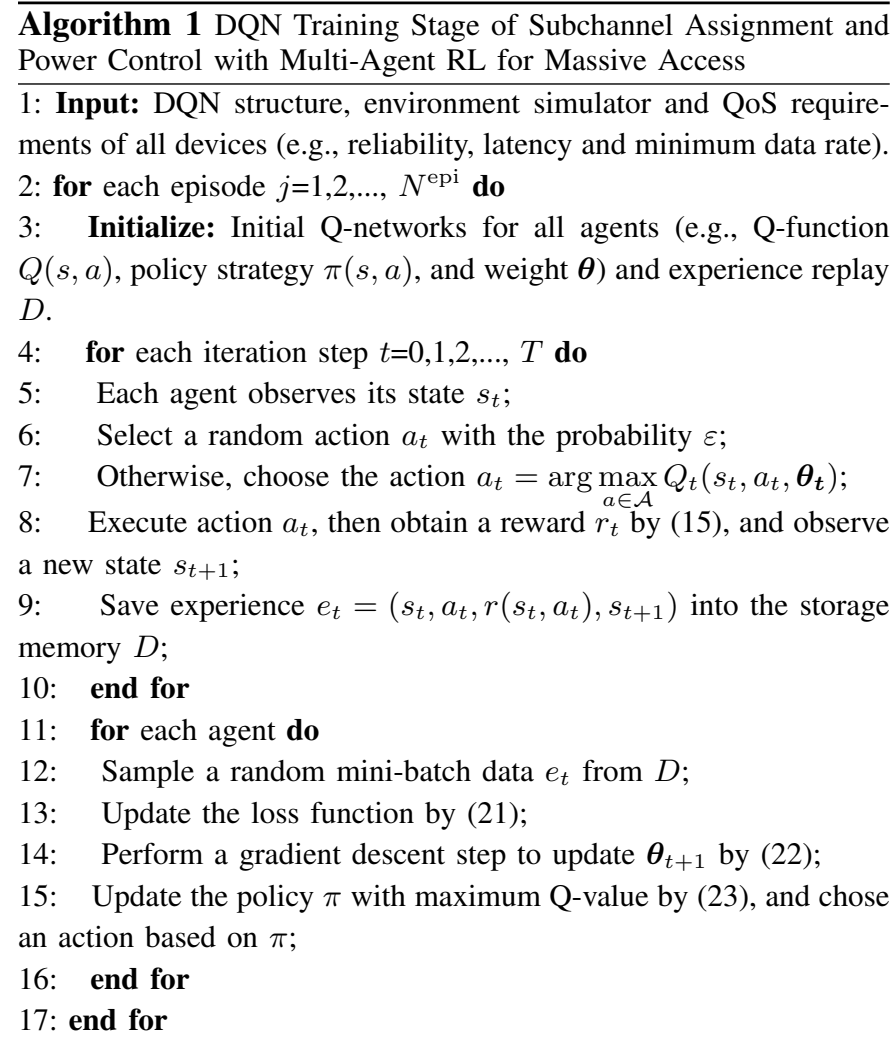

18: return: Return trained DQN models.

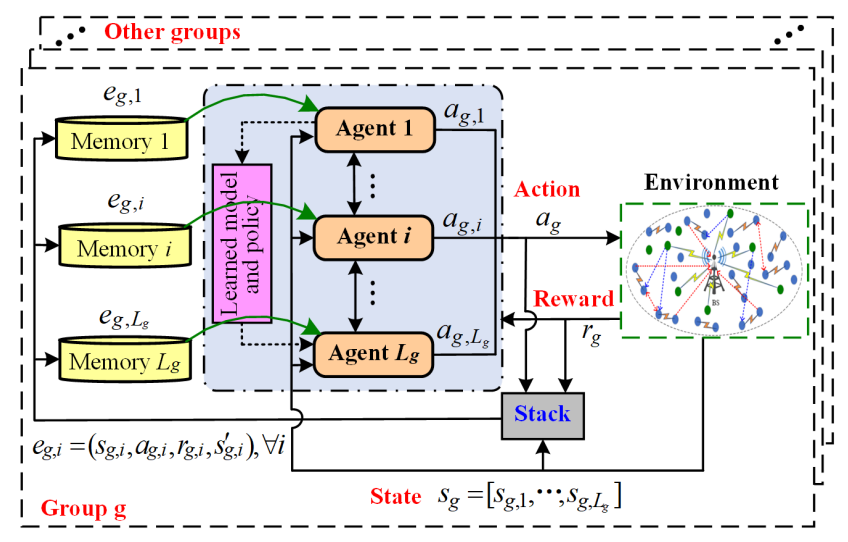

Fig. 3. Distributed cooperative multi-agent RL framework.

their own learning model with extra training computational time or cost. In order to address this issue, motivated by the concept of transfer learning and cooperative learning, we present a distributed cooperative learning approach to improve the learning efficiency and enhance the service performance of each agent, where devices are encouraged to communicate and share their learned experiences and decisions within a small number of neighbors, and finally learn with each other, as shown in Fig.3.

1) Transfer Learning:

(i) The Expert Agent Selection: When a new device joins $5 \mathrm{G}$ and B5G networks, or one device applies a new communication service, instead of building a new learning model, it can communicate with neighboring devices to search one suitable expert to utilize the expert' current learning model. In addition, if one communication link has poor performance (e.g., low convergence speed and poor QoS satisfaction levels) according to its current leaning strategy, it can search one neighboring communication link (agent) as the expert and then utilizes the learned model or policy from the expert.

Generally, to find the expert, devices exchange the following several metrics with their neighbors: $a$ ) the types of device, e.g., C-device and D2D device; $b$ ) the communication services, which mainly refer to URLLC service and normal service; $c$ ) the related QoS parameters, such as the target thresholds of reliability and latency, and the minimum data rate. The similarity of the agents can be evaluated by adopting the manifold learning, which is also called Bregman Ball [19]. The Bregman Ball is defined as the minimum manifold with a central $\Theta_{\text {cen }}$ (the information of the learning agent, where information refers to the types of device,communication services, and QoS parameters mentioned above), and a radius $\Psi_{\text {rad }}$. Any information point $\Theta_{\text {poi }}$ (the information of neighbors) is inside this ball, and the agent tries to search the information point which has the highest similarity with $\Theta_{\text {cen }}$. The distance between any information point and the central $\Theta_{\text {cen }}$ is defined by

$$
\operatorname{Dis}\left(\Theta_{\mathrm{cen}}, \Psi_{\mathrm{rad}}\right)=\left\{\Theta_{\text {poi }} \in \Theta: \operatorname{Dis}\left(\Theta_{\mathrm{poi}}, \Theta_{\mathrm{cen}}\right) \leq \Psi_{\mathrm{rad}}\right\} .
$$

After the highest similarity level (the smallest distance achieved by (24)) between the learning agent and the expert agent is found, the learning agent can use the learned DQN model of the selected expert agent.

(ii) Learning from Expert Agent: As analyzed above, after finding the expert agent, the learning agent uses the transferred DQN model $Q^{\text {Transfer }}(s, a)$ from the expert agent and its current native DQN model $Q^{\text {Current }}(s, a)$ to generate an overall DQN model. Accordingly, the new Q-table of the learning agent can be expressed as

$$
Q^{\text {New }}(s, a)=\mu Q^{\text {Transfer }}(s, a)+(1-\mu) Q^{\text {Current }}(s, a),
$$

where $\mu \in[0,1]$ is the transfer rate, and it will be gradually decreased after each learning step to reduce the effect of the transferred DQN model from the expert agent on the new DQN model.

In the distributed cooperative manner, the policy vector of all agents are updated as follows:

$\boldsymbol{\pi}_{t+1}\left(s_{t}\right)=\left[\begin{array}{c}\pi_{t+1}^{1}\left(s_{t}^{1}\right) \\ \vdots \\ \pi_{t+1}^{i}\left(s_{t}^{i}\right) \\ \vdots \\ \pi_{t+1}^{Z}\left(s_{t}^{Z}\right)\end{array}\right]=\left[\begin{array}{c}\arg \max _{a^{1} \in \mathcal{A}^{1}}\left\{Q_{t+1}^{1}\left(s_{t}^{1}, a_{t}^{1}\right)\right\} \\ \vdots \\ \arg \max _{a^{i} \in \mathcal{A}^{i}}\left\{Q_{t+1}^{i}\left(s_{t}^{i}, a_{t}^{i}\right)\right\} \\ \vdots \\ \arg \max _{a^{Z} \in \mathcal{A}^{Z}}\left\{Q_{t+1}^{Z}\left(s_{t}^{Z}, a_{t}^{Z}\right)\right\}\end{array}\right]$

where $Q_{t+1}^{i}\left(s_{t}^{i}, a_{t}^{i}, \boldsymbol{\theta}_{t}^{i}\right)$ denotes the Q-function of the $i$-th agent (communication link) with its current state-action pair $\left(s_{t}^{i}, a_{t}^{i}\right)$ at the current time slot in its DQN model. 
When the state-action pairs are visited for many enough times for convergence, all Q-tables will converge to the final point $Q^{*}$. Hence, we can get the final learned policy as follow

$$
\boldsymbol{\pi}^{*}(s)=\left[\begin{array}{c}
\arg \max _{a^{1} \in \mathcal{A}^{1}}\left\{Q^{1^{*}}\left(s^{1}, a^{1}\right)\right\} \\
\vdots \\
\arg \max _{a^{Z} \in \mathcal{A}^{Z}}\left\{Q^{Z^{*}}\left(s^{Z}, a^{Z},\right)\right\}
\end{array}\right] \text {. }
$$

2) Cooperative Learning

If the action is chosen independently according to the local information, each communication link has no information of actions selected by other communication links when the actions are updated simultaneously. Consequently, the states observed by each communication link may fail to fully characterize the environment. Hence, cooperation and decision sharing among agents in the proposed distributed learning approach can improve the network performance, where a small number of communication links will share their actions with their neighbors. In the cooperative manner, the massive number of agents can be classified into $G$ groups, where the $g$-th group consists of $L_{g}$ agents and the agents in the same group are also their neighboring agents. The group division principle can adopt the studies in [13], [26] and [32].

In general, it is possible to approximate the sum utility of the $g$-th group $Q_{g}\left(s_{g}, a_{g}\right)$ by the sum of each agent' utility $Q_{g, i}\left(s_{g, i}, a_{g, i}\right)$ in the same group, where $s_{g}$ and $a_{g}$ denote the entire state and action of the $g$-th group, respectively; $s_{g, i}$ and $a_{g, i}$ are the individual state and action of the $i$-th agent in the $g$-th group, respectively. Hence, the total utility in a small group $g$ can be calculated by

$$
Q_{g}\left(s_{g}, a_{g}\right)=\sum_{i=1}^{L_{g}} Q_{g, i}\left(s_{g, i}, a_{g, i}\right) .
$$

Then, the joint optimal policy learned in the $g$-th group can be expressed by

$$
\pi_{g}\left(s_{g}\right)=\arg \max _{a_{g} \in \mathcal{A}_{g}}\left\{Q_{g}\left(s_{g}, a_{g}\right)\right\},
$$

where $\mathcal{A}_{g}$ denotes the entire action space of the $g$-th group.

In fact, the cooperation can be defined by allowing communication links (agents) to share their selected actions with their neighboring links and take turns to make decisions, which can enhance the overall feedback reward by choosing the actions jointly instead of independently. For example, in the fully distributed learning manner, each spectrum access may run into collisions when other links make their decisions independently and happen to assign the same subchannel, leading to the increased co-channel interference and reduce the performance. By contrast, in the cooperative learning scenario, to avoid such situation, each communication link has information of the neighbors' actions in its observation, and try to avoid the assignment of the same subchannel in order to achieve more rewards.

The distributed cooperative implementation of multi-agent RL for massive access is shown in Algorithm 2. Generally, at each time step, after observing the states (subchannel occupation status, channel quality, traffic load, QoS satisfaction level, etc.) from the environment, the actions (massive subchannel assignment and power control) in communication links are

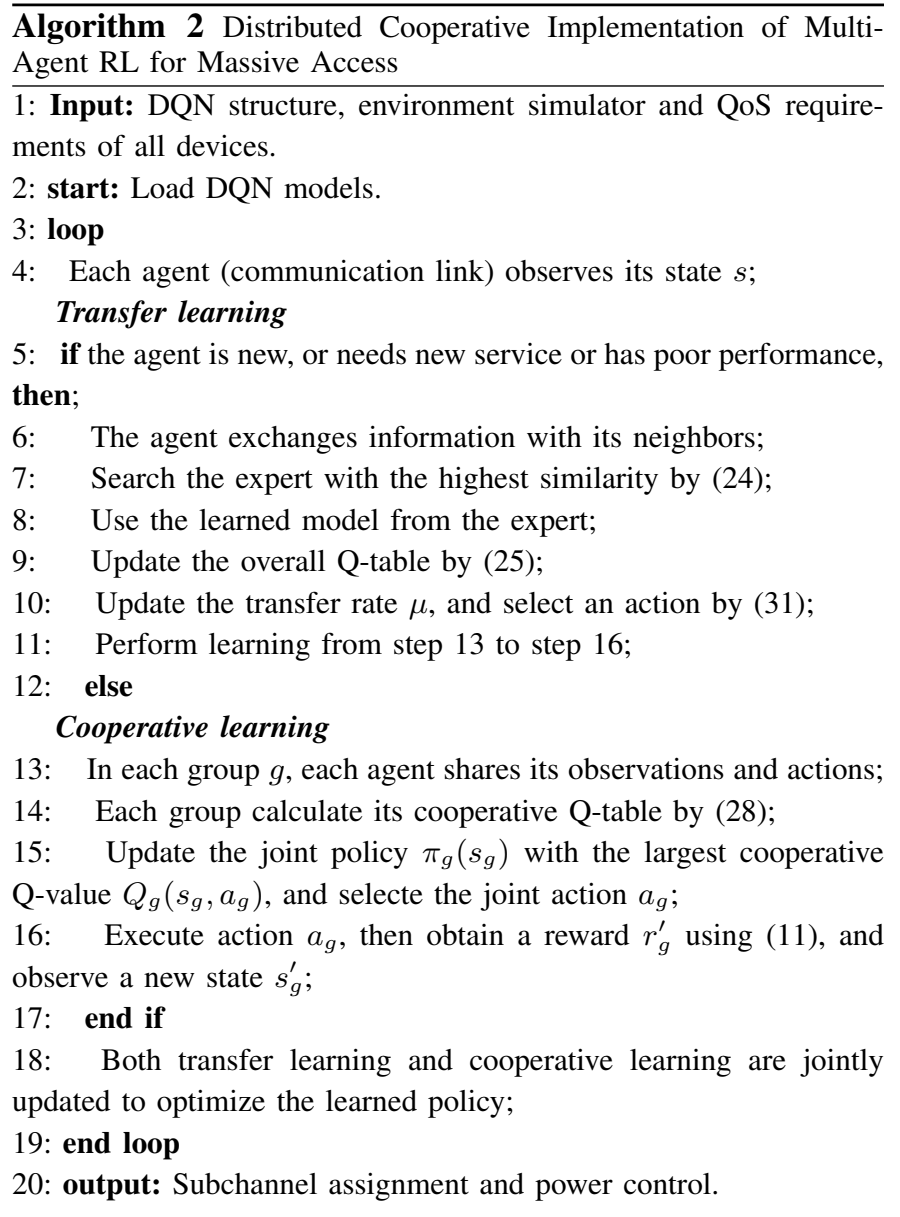

selected with the maximum Q-value given by loading the trained DQN models shown in Algorithm 1. As mentioned above, a small number of neighboring devices are encouraged to cooperate with each other in the same group to maximize the sum Q-value shown in (28), where their decisions are shared in the same group and the joint action strategy $a_{g}$ is selected with the maximum cooperative Q-value. In addition, it is worth noting that if a new device joins the network or applies a new service, or one communication link achieves poor performance (e.g, low transmission success probability or low convergence speed), then it can directly search the expert agent from the neighbors in the same group, and utilizes the transfer learning model and policy from the expert agent. Finally, all communication links begin transmission with the subchannel assignment and transmission power strategies determined by their learned policies.

Multi-agent reinforcement learning is also called "independent DQN", where each agent independently learns its own policy and considers other agents as part of the environment. Moreover, , the combination of experience replay with independent DQN appears to be problematic: the nonstationarity introduced by independent DQN. Hence, we have presented a distributed cooperative multi-agent DQN scheme, devices are encouraged to communicate and share their learned experiences and actions within a small number of neighbors, and finally learn with each other. In this case, the scheme 
is capable of avoiding the non-stationarity of independent Qlearning by having each agent learn a policy that conditions on an information-sharing of the other agents' policies (behaviors) in the same group.

\section{Computational Complexity Analysis}

For the training phase, in trained DQN models, let $L, B_{0}$ and $B_{l}$ denote the training layers which are proportional to the number of states, the size of the input layer and the number of neurons used in DQN, respectively. The complexity in each time step for each agent is calculated by $O\left(B_{0} B_{1}+\sum_{l=1}^{L-1} B_{l} B_{l+1}\right)$ at each training step. In the training phase, each mini-batch has episodes $N^{\text {epi }}$ with each episode being $T$ time steps, and each trained model is completed over $I$ iterations until convergence and the network has $Z$ agents with the $Z$ trained DQN models. Hence, the total computational complexity is $O\left(Z I N^{\mathrm{epi}} T\left(B_{0} B_{1}+\sum_{l=1}^{L-1} B_{l} B_{l+1}\right)\right)$. The high computational complexity of the DQN training phase can be performed offline for a finite number of episodes at a powerful unit (such as the BS) [38], [39].

For the distributed cooperative phase (also called testing phase), our proposed approach applies the transfer learning mechanism and allows the expert agent to share the learned knowledge or actions with other agents. Let $\mathcal{S}^{\prime}$ and $\mathcal{A}^{\prime}$ denote the stored state space and action space, respectively. The computational complexity of the classical DQN approach (the fully distributed DQN approach) and the proposed approach are $O\left(|S|^{2} \times|\mathcal{A}|\right)$ and $O\left(\left|\mathcal{S}^{\prime}\right|^{2} \times\left|\mathcal{A}^{\prime}\right|+|\mathcal{S}|^{2} \times|\mathcal{A}|\right)$ [19], respectively, indicating that the complexity of the proposed approach is higher than the classical DQN learning approach. Nevertheless, the stored state space and action space in the memory is not large at each device, and hence the complexity of the proposed learning approach is slightly higher than the classical DQN approach. For cooperative learning, a small number of agents in each same group will select their actions jointly instead of independently by sharing their own selected action. Let $a_{g, i}^{c o}$ denote the shared action set of each $i$-th agent in the $g$-th group in the current time slot, then the computational complexity the $g$-th group in term of action sharing is $O\left(\sum_{i=1}^{L_{g}}\left|a_{g, i}^{c o}\right|\right)$. As the network has $G$ groups, the total computational complexity of the cooperative learning is $O\left(\sum_{g=1}^{G} \sum_{i=1}^{L_{g}}\left|a_{g, i}^{c o}\right|\right)$.

\section{Simulation Results and Analysis}

In this section, simulation results are provided to evaluate the proposed distributed cooperative multi-agent RL based massive access approach. We consider a single cell with a cell radius of $500 \mathrm{~m}$, the total number of devices is 2000 . In addition, we set one fifth of the total number of devices to be normal services and the minimum data rate requirement is set as $3.5 \mathrm{bps} / \mathrm{Hz}$. The maximum D2D communication distance is $75 \mathrm{~m}$. The carrier frequency is $2 \mathrm{GHz}$, and the total bandwidth is $100 \mathrm{MHz}$ which is equally divided into 100 subchannels with each subchannel having $1 \mathrm{MHz}$. For the URLLC services, the SINR threshold is $5 \mathrm{~dB}$, the processing/computing delay $T_{\mathrm{pc}}=0.3 \mathrm{~ms}$, the reliability requirement varies between $99.9 \%$ and $99.99999 \%$, and the maximum latency threshold varies
TABLE I

Simulation PARAMETERS

\begin{tabular}{|l|l|}
\hline Parameters & Value \\
\hline Cell radius & $500 \mathrm{~m}$ \\
\hline Carrier frequency, bandwidth & $2 \mathrm{GHz}, 100 \mathrm{MHz}$ \\
\hline Maximum D2D communication distance & $75 \mathrm{~m}$ \\
\hline Total number of devices & 2000 \\
\hline Number of subchannels & 100 \\
\hline Reliability requirement in URLLC links & $99.9 \%, 99.99 \%, \ldots, 99.99999 \%$ \\
\hline Latency threshold & $1,2,4,6,8,10 \mathrm{~ms}$ \\
\hline Minimum capacity of each normal link & $3.5 \mathrm{bps} / \mathrm{Hz}$ \\
\hline SINR threshold in URLLC links & $5 \mathrm{~dB}$ \\
\hline Maximum transmit power of each device & $500 \mathrm{~mW}$ \\
\hline The circuit power consumption of each device & $50 \mathrm{~mW}$ \\
\hline Background noise power & $-114 \mathrm{dBm}$ \\
\hline Each packet size in URLLC links & $1024 \mathrm{bytes}$ \\
\hline
\end{tabular}

between $1 \mathrm{~ms}$ and $10 \mathrm{~ms}$ for different simulation settings. The maximum transmit power of each device and circuit power consumption are $500 \mathrm{~mW}$ and $50 \mathrm{~mW}$, respectively. The background noise power is $-114 \mathrm{dBm}$. Each packet size in URLLC links is 1024 bytes. The DQN model consists of three connected hidden layers, containing 250, 250, and 100 neurons, respectively. The learning rate is $\alpha=0.02$ and discount factor is set to be $\gamma=0.95$.

We compare the proposed distributed cooperative multiagent RL based massive access approach (denoted as proposed DC-DRL MA, which adopts both transfer learning and cooperative learning mechanisms) with the following approaches:

1) The group based massive access approach, where devices are grouped by the similarities with each group having one group leader to communicate with the centralized controller. Then, the subchannel assignment and transmission power control are adjusted iteratively to the communication links in each group, similar to the group based preamble reservation access approach [13] (denoted as centralized G-MA).

2) The fully distributed multi-agent RL based massive access approach (denoted as fully D-DRL MA [37]), similar to the approach [37], where each communication link selects its subchannel assignment and transmission power strategy based on its own local information without cooperating with other communication links.

3) Random massive access approach (denoted as random MA), where each communication link chooses its subchannel assignment and transmission power strategy in a random manner.

\section{A. Convergence Comparisons}

Here, we show in Fig. 4 the energy efficiency (EE) with increasing training episodes to investigate the convergence behavior of the proposed multi-agent DQN approach and compared approaches. Clearly, the proposed learning approach significantly achieves the higher EE performance than that of the fully distributed DRL approach [37] and random MA 


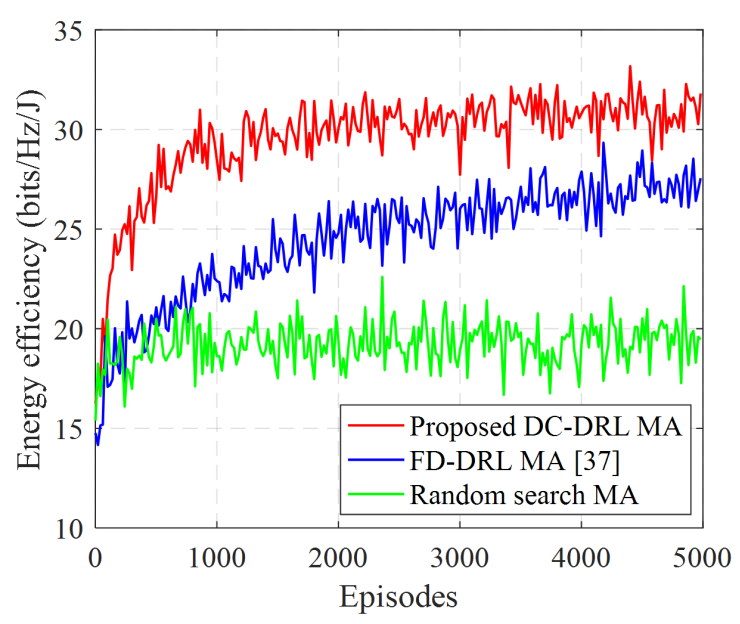

Fig. 4. Convergence comparisons of compared learning approaches.

approach. Especially, the proposed approach has faster convergence speed and less fluctuations by adopting by transfer learning and cooperative learning mechanisms to improve the learning efficiency and convergence speed. The fully distributed DRL approach [37] is simple without any cooperation among devices, but it achieves poor global performance, leading to the poor EE value. Even though the random MA approach has the simplest structure, the worst performance fails to optimize the network energy efficiency with increasing training episodes. Our proposed approach applies both the transfer learning and cooperative learning mechanisms to enhance the convergence speed and learning efficiency, and the optimized strategy can be learned after a number of training episodes. From Fig. 4 , the energy efficiency per episode improves as training continues, demonstrating the effectiveness of the proposed training approach. When the training episode approximately reaches 1900, the performance gradually converges despite some fluctuations due to mobility-induced channel fading in mobile environments. Since we investigate the resource management in massive access scenario, the environment is complex, as well as the action and state spaces are large for all mobile devices, so our presented learning approach requires about 2000 training episodes to appropriately converge.

\section{B. Performance Comparisons Under Different Thresholds of Reliability and Latency}

Fig. 5 and Fig. 6 compare the performances of all approaches under different values of the reliability and latency thresholds, respectively, when the packet arrival rate is 0.03 packets/slot/per link and the total number of devices is 2000 . From both Fig. 5 and Fig. 6, for all approaches, we can find that both the EE performance and the transmission success probability drop as the required reliability value increases and the maximum latency threshold decreases. The reason is that the more stringent the reliability and latency constraints are, the worse network EE and transmission success probability the network can archive. In this case, both the transmission power and subchannel assignment strategy needs to be carefully designed to guarantee the stringent reliability and latency
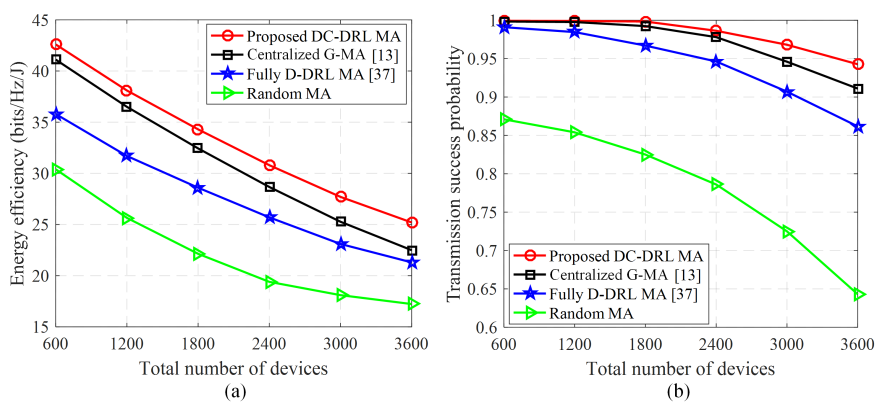

Fig. 5. Performance comparisons vs. different reliability thresholds.
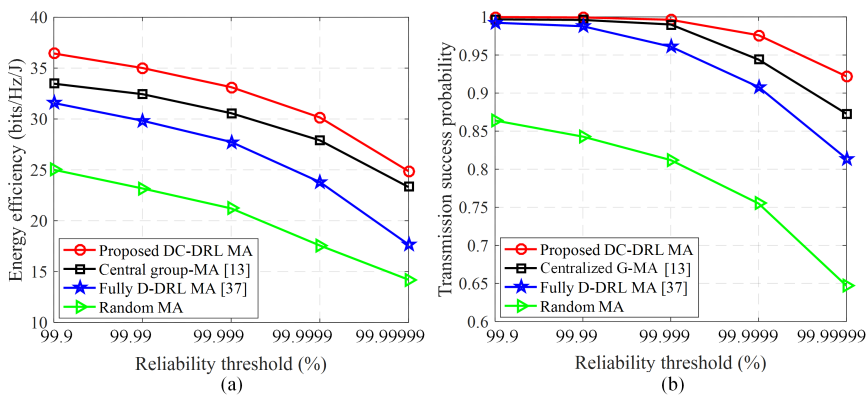

Fig. 6. Performance comparisons vs. different latency thresholds.

constraints, such that the transmission success probability can be guaranteed at a high level.

We also observe from Fig. 5 (b) and Fig. 6 (b) that within a reasonable region of the reliability and latency thresholds change, the three approaches (except the random search approach) can till achieve the high transmission success probability, which, however, have more unsatisfied transmission link events happen if the constraints are extremely stick (e.g., the reliability threshold grows beyond $99.999 \%$ or the maximum latency threshold is less than $4 \mathrm{~ms}$ ). Compared with other approaches, our proposed approach achieves the higher EE performance and transmission success probability under different reliability and latency requirements, especially the performance gap between the proposed approach and other approaches becomes more significant when the constraints become more stringent. The reason is that our proposed approach employs both the transfer learning and cooperative learning mechanisms to optimize the global subchannel assignment and transmission power strategy, thereby improving the network performance. From Fig. 5 (a) and Fig. 6 (a), an interesting observation is that compared with the centralized G-MA approach [13] and random MA approach, the EE value curve declines more quickly in our proposed approach when the constraints become stricter. The reason is that the proposed approach designs the specific QoS-aware reward function shown in (15) to try to guarantee QoS requirements (meeting the high transmission success probability), and hence the network may sacrifice the part of EE performance to support more successful transmission communication links. 


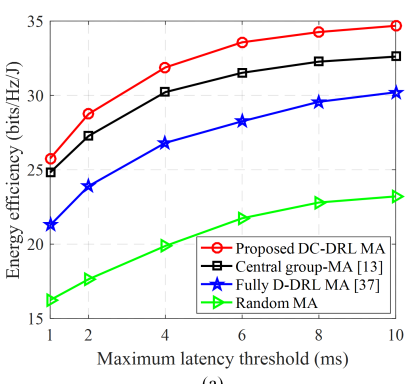

(a)

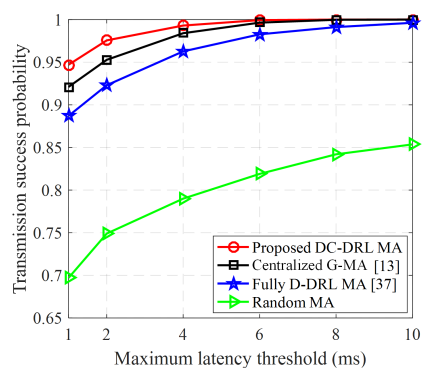

(b)
Fig. 7. Performance comparisons with different packet arrival rates.

\section{Performance Comparisons Versus Packet Arrival Rate}

Fig. 7 represents the performance comparisons with respect to the increasing packet arrival rate for different massive access approaches, when the number of devices is 2000 , and the reliability and latency thresholds are $99.999 \%$ and $5 \mathrm{~ms}$, respectively. From Fig. 7, with the growing packet arrival rate, both the EE performance and the transmission success probability decrease slightly for all approaches when the packet arrival rate is less than a certain threshold, but drop sharply when the packet arrival rate grows beyond the acceptable margin. An increase of packet arrival rate results in longer transmission duration (e.g., transmission delay and queue waiting delay), frequent spectrum access and possibly increases more transmission power in order to improve packet transmission success probability. In addition, the increase of packet arrival rate also leads to stronger co-channel interference for a longer period, which limits the data rate improvement. Hence, as shown in Fig. 7 (a), the EE performance decreases slightly with the increase of packet arrival rate when packet arrival rate is not high, and the performance will become worse if packet arrival rate grows beyond the acceptable margin.

From Fig. 7 (b), even though the transmission success probability drops for all approaches, the proposed approach still achieves the better performance than other three approaches. Remarkably, the proposed approach attains approximately $100 \%$ success transmission probability when the packet arrival rate is less than 0.03 packets per time slot, and achieves noticeable degradation when the packet size grows beyond 0.03 packets per time slot. Such the performance degradation may result from the limited spectrum resource, where the current subchannel resource cannot completely support the massive number of transmission packets simultaneously with the increasing packet arrival rate.

\section{CONCLUSION}

In this paper, a distributed cooperative channel assignment and power control approach based on multi-agent RL has been presented to solve the massive access management problem in future wireless networks, where the proposed approach is capable of supporting different QoS requirements (e.g., URLLC and minimum data rate) of a huge number of devices. The proposed multi-agent RL based approach consists of a centralized training procedure and a distributed cooperative implementation procedure. In order to improve the network performance and QoS satisfaction levels, the transfer learning and cooperative learning mechanisms have been employed to enable communication links to work cooperatively in a distributed cooperative way. Simulation results have confirmed the effectiveness of the proposed learning approach and also showed that the proposed approach outperforms other existing approaches in massive access scenario.

\section{REFERENCES}

[1] M. R. Palattella, et al., "Internet of Things in the 5G era: Enablers, architecture, and business models," IEEE J. Sel. Areas Commun., vol. 34, no. 3, pp. 510-527, Mar. 2016.

[2] A. I. Sulyman, S. M. A. Oteafy, and H. S. Hassanein, "Expanding the cellular-IoT umbrella: An architectural approach," IEEE Wireless Commun., vol. 24, no. 3, pp. 66-71, Jun. 2017.

[3] L. Liu, E. G. Larsson, W. Yu, P. Popovski, C. Stefanovic, and E. de Carvalho, "Sparse signal processing for grant-free massive connectivity: A future paradigm for random access protocols in the Internet of Things," IEEE Signal Procss. Mag., vol. 35, no. 5, pp. 88-99, Sept. 2018.

[4] P. Popovski, "Ultra-reliable communication in 5G wireless systems," in Proc. 1st Int. Conf. 5 G Ubiquitous Connectivity, pp. 146-151, Nov. 2014

[5] N. Jiang, Y. Deng, A. Nallanathan, X. Kang, and T. Q. S. Quek, "Analyzing random access collisions in massive IoT networks," IEEE Trans. Wireless Commun., vol. 17, no. 10, pp. 6853-6870, Oct. 2018.

[6] S. Y. Jung, S. H. Lee, and J. Kim, "Reliability control framework for random access of massive IoT Devices," IEEE Access, vol. 7, pp. 4992849937, 2019.

[7] R. Cheng, Z. Becvar, Y. Huang, G. Bianchi, and R. Harwahyu, "Twophase random access procedure for LTE-A networks," IEEE Trans. Wireless Commun., vol. 18, no. 4, pp. 2374-2387, Apr. 2019.

[8] Y. Liu, C. Yuen, X. Cao, N. U. Hassan, and J. Chen, "Design of a scalable hybrid MAC protocol for heterogeneous M2M networks," IEEE Internet Things J., vol. 1, no. 1, pp. 99-111, Feb. 2014.

[9] T. Ding, X. Yuan, and S. C. Liew, "Sparsity learning-based multiuser detection in grant-free massive-device multiple access," IEEE Trans. Wireless Commun., vol. 18, no. 7, pp. 3569-3582, Jul. 2019.

[10] P. Wang, B. Di, H. Zhang, K. Bian, and L. Song, "Cellular V2X communications in unlicensed spectrum: Harmonious coexistence with VANET in 5G systems," IEEE Trans. Wireless Commun., vol. 17, no. 8, pp. 5212-5224, Aug. 2018.

[11] "Study on RAN improvements for machine-type communications, v11.0.0," 3GPP, Sophia Antipolis, France, Rep. TR 37.868, Sep. 2011.

[12] M. Lee, Y. Kim, Y. Piao, and T. Lee, "Recycling random access opportunities with secondary access class barring," IEEE Trans. Mobile Comput., vol. 19, no. 9, pp. 2189-2201, 1 Sept. 2020.

[13] T. N. Weerasinghe, I. A. M. Balapuwaduge, and F. Y. Li, "Preamble reservation based access for grouped mMTC devices with URLLC requirements," in Proc. IEEE ICC, Shanghai, China, 2019, pp. 1-6.

[14] Z. Shi, X. Xie, and H. Lu, "Deep reinforcement learning based intelligent user selection in massive MIMO underlay cognitive radios," IEEE Access, vol. 7, pp. 110884-110894, 2019.

[15] P. Popovski et al., "Wireless access in ultra-reliable low-latency communication (URLLC)," IEEE Trans. Commun., vol. 67, no. 8, pp. 57835801, Aug. 2019.

[16] L. Zhao, X. Chi, L. Qian, and W. Chen, "Analysis on latency-bounded reliability for adaptive grant-free access with multi-packets reception (MPR) in URLLCs," IEEE Commun. Lett., vol. 23, no. 5, pp. 892-895, May 2019.

[17] S. Doğan, A. Tusha, and H. Arslan, "NOMA with index modulation for uplink URLLC through grant-free access," IEEE J. Sel. Topics in Signal Process., vol. 13, no. 6, pp. 1249-1257, Oct. 2019.

[18] S. E. Elayoubi, P. Brown, M. Deghel, and A. Galindo-Serrano, "Radio resource allocation and re transmission schemes for URLLC over $5 \mathrm{G}$ networks," IEEE J. Sel. Areas Commun., vol. 37, no. 4, pp. 896-904, Apr. 2019.

[19] H. Yang, A. Alphones, W. Zhong, C. Chen, and X. Xie, "LearningBased energy-efficient resource management by heterogeneous RF/VLC for ultra-reliable low-latency industrial IoT networks," IEEE Trans. Ind. Informat., vol. 16, no. 8, pp. 5565-5576, Aug. 2020.

[20] X. Chen, Z. Zhang, C. Zhong, D. W. K. Ng, and R. Jia, "Exploiting interuser interference for secure massive non-orthogonal multiple access," IEEE J. Sel. Areas Commun., vol. 36, no. 4, pp. 788-801, Apr. 2018. 
[21] G. Yu, X. Chen, and D. W. K. Ng, "Low-cost design of massive access for cellular Internet of Things," IEEE Trans. Commun., vol. 67, no. 11, pp. 8008-8020, Nov. 2019.

[22] I. Budhiraja, S. Tyagi, S. Tanwar, N. Kumar, and J. J. P. C. Rodrigues, "Tactile internet for smart communities in 5G: An insight for NOMAbased solutions," IEEE Trans. Ind. Informat., vol. 15, no. 5, pp. 31043112, May 2019.

[23] Y. Han, B. D. Rao, and J. Lee, "Massive uncoordinated access with massive MIMO: A dictionary learning approach," IEEE Trans. Wireless Commun., vol. 19, no. 2, pp. 1320-1332, Feb. 2020.

[24] Z. Chen, F. Sohrabi, and W. Yu, "Multi-cell sparse activity detection for massive random access: Massive MIMO versus cooperative MIMO," IEEE Trans. Wireless Commun., vol. 18, no. 8, pp. 4060-4074, Aug. 2019.

[25] H. Zhang, Y. Liao, and L. Song, "D2D-U: Device-to-device communications in unlicensed bands for 5G system," IEEE Trans. Wireless Commun., vol. 16, no. 6, pp. 3507-3519, Jun. 2017.

[26] M. Gharbieh, A. Bader, H. ElSawy, H. Yang, M. Alouini, and A. Adinoyi, "Self-organized scheduling request for uplink $5 \mathrm{G}$ networks: A D2D clustering approach," IEEE Trans. Commun., vol. 67, no. 2, pp. 1197-1209, Feb. 2019.

[27] L. Liu and W. Yu, "A D2D-based protocol for ultra-reliable wireless communications for industrial automation," IEEE Trans. Wireless Commun., vol. 17, no. 8, pp. 5045-5058, Aug. 2018.

[28] H. Yang, X. Xie, and M. Kadoch, "Intelligent resource management based on reinforcement learning for ultra-reliable and low-latency IoV communication networks," IEEE Trans. Veh. Technol., vol. 68, no. 5, pp. 4157-4169, May 2019.

[29] S. Han et al., "Energy efficient secure computation offloading in NOMAbased mMTC networks for IoT," IEEE Internet Things J., vol. 6, no. 3, pp. 5674-5690, Jun. 2019.

[30] R. Mahapatra, Y. Nijsure, G. Kaddoum, N. U. Hassan, and C. Yuen, "Energy efficiency tradeoff mechanism towards wireless green communication: A survey," IEEE Commun. Surveys Tuts., vol. 18, no. 1, pp. 686-705, 1st 2016.

[31] D. Zhai, R. Zhang, L. Cai, B. Li, and Y. Jiang, "Energy-efficient user scheduling and power allocation for NOMA-based wireless networks with massive IoT devices," IEEE Internet Things J., vol. 5, no. 3, pp. 1857-1868, Jun. 2018.

[32] G. Miao, A. Azari, and T. Hwang, " $E^{2}$-MAC: energy efficient medium access for massive M2M communications," IEEE Trans. Commun., vol. 64, no. 11 , pp. 4720-4735, Nov. 2016

[33] Y. Teng, M. Yan, D. Liu, Z. Han, and M. Song, "Distributed learning solution for uplink traffic control in energy harvesting massive machinetype communications," IEEE Wireless Commun. Lett., vol. 9, no. 4, pp. 485-489, Apr. 2020

[34] T. Park and W. Saad, "Distributed learning for low latency machine type communication in a massive Internet of Things," IEEE Internet Things J., vol. 6, no. 3, pp. 5562-5576, Jun. 2019.

[35] Y. Ruan, W. Wang, Z. Zhang, and V. K. N. Lau, "Delay-aware massive random access for machine-type communications via hierarchical stochastic learning," in Proc. IEEE ICC, Paris, 2017, pp. 1-6.

[36] C. Di, B. Zhang, Q. Liang, S. Li, and Y. Guo, "Learning automata-based access class barring scheme for massive random access in machine-tomachine communications," IEEE Internet Things J., vol. 6, no. 4, pp. 6007-6017, Aug. 2019.

[37] H. Chang, H. Song, Y. Yi, J. Zhang, H. He, and L. Liu, "Distributive dynamic spectrum access through deep reinforcement learning: A reservoir computing-based approach," IEEE Internet Things J., vol. 6, no. 2, pp. 1938-1948, Apr. 2019.

[38] O. Naparstek and K. Cohen, "Deep multi-user reinforcement learning for distributed dynamic spectrum access," IEEE Trans. Wireless Commun., vol. 18, no. 1, pp. 310-323, Jan. 2019.

[39] L. Liang, H. Ye, and G. Y. Li, "Spectrum sharing in vehicular networks based on multi-agent reinforcement learning," IEEE J. Sel. Areas Commun., vol. 37, no. 10, pp. 2282-2292, Oct. 2019.

[40] Y. Hua, R. Li, Z. Zhao, X. Chen, and H. Zhang, "GAN-powered deep distributional reinforcement learning for resource management in network slicing," to appear in IEEE J. Sel. Areas Commun., vol. 38, no. 2, pp. 334-349, Feb. 2020.

[41] Y. Yu, T. Wang, and S. C. Liew, "Deep-reinforcement learning multiple access for heterogeneous wireless networks," IEEE J. Sel. Areas Commun., vol. 37, no. 6, pp. 1277-1290, Jun. 2019.

[42] F. Shah-Mohammadi and A. Kwasinski, "Deep reinforcement learning approach to QoE-driven resource allocation for spectrum underlay in cognitive radio networks," in Proc. IEEE ICC Workshops, Kansas City, MO, 2018, pp. 1-6.
[43] Y. Jiang, "Network calculus and queueing theory: Two sides of one coin," in Proc. 4th Int. ICST Conf. Perform. Eval. Methodologies Tools, Brussels, Belgium, 2009, pp. 1-11. 